\section{Ensuring safe blood in the Americas}

\author{
George A. O. Alleyne 1
}

It is a pleasure to write an introductory statement for this special issue of the Revista Panamericana de Salud Pública/Pan American Journal of Public Health devoted to blood banks. This issue will deal not only with blood banks as the places where blood is collected and processed, but also with the whole spectrum of transfusion medicine. Blood has a special place in the culture of our civilizations as symbolizing strength and the vital force of life. Its use in forms that may seem macabre to us now found its way into many ancient rituals. All physicians regard blood with some awe since so far there is no substitute for it, and its transfusion may be regarded as the first organ transplant. The history of transfusion and some of the mystique surrounding it have been set out vividly in one of the issues of Perspectives, the news magazine of the Pan American Health Organization (PAHO). I was amused by one of the special historical vignettes related to blood transfusion. The year 1492 is remembered as the year of the meeting of the two worlds, and it is less remembered as the year of perhaps the first transfusion. It is said that in that year Pope Innocent VIII was transfused for a neurological illness, but the therapy was rather ineffective, as the worthy prelate died. Fortunately, recent history deals with transfusion more kindly.

Blood transfusion and blood banks have been objects of PAHO's technical cooperation for many years. The basic focus has been on the screening of blood to prevent transmission of disease, and this was seen as falling predominantly within the technical area of laboratory services. The section on blood banks in PAHO's Health in the Americas quadrennial publication from 1998 deals almost exclusively with the number of units of blood collected and the testing done to prevent disease transmission.

At my reelection as Director of PAHO in 1998 I challenged the Organization to work towards making all the blood in the Region of the Americas safe, and PAHO's Strategic and Programmatic Orientations, 1999-2002 referred specifically to having our technical cooperation "supporting improved safety and quality in blood bank operations." When the PAHO Governing Bodies considered the topic in 1999, they approved a resolution urging Member
Dr. Alleyne wrote this shortly before completing his term as Director of the Pan American Sanitary Bureau on 31 January 2003. 
States to "give higher priority in their national health policies to the safety of blood for transfusion." The resolution also emphasized the development of national blood programs and transfusion services based on voluntary, nonremunerated blood donation.

"I wish I could say that the challenge of having all the blood in the Americas safe has been met. It has not, but there has been significant progress."

Fortuitously, the year 2000 saw two major events that gave a fillip to our work in this area. World Health Day 2000 had as its theme "safe blood starts with me - safe blood saves lives," and a great deal of enthusiasm was generated for providing safe blood. A generous grant from the Bill and Melinda Gates Foundation allowed PAHO to intensify its efforts in the area, and in that same year PAHO launched its Regional Safe Blood Initiative. I wish I could say that the challenge of having all the blood in the Americas safe has been met. It has not, but there has been significant progress.

In my view, one of the very significant advances has been in the conceptualization of the role of transfusional medicine in public health. For many years I had the impression that the focus for blood was on its clinical use and that transfusional medicine was concerned primarily with the proper use of blood and blood products. Perhaps the increased awareness of the possibility of transmitting diseases such as HIV/AIDS, Chagas' disease, and the various forms of hepatitis has elevated the matter of the proper collection, screening, and use of blood into the realm of public health. This may be a good example of the dictum that the health of the public individually or collectively is not the province of any single discipline or area of specialization.

I am pleased at the progress that is being made. There is a proper thrust to have all blood come from nonremunerated voluntary donors, as is the practice in Aruba, Canada, Cuba, Curaçao, and the United States of America. The promotion of this practice is supported by the evidence of increased incidence of infection when blood is not collected in this manner. The program to increase nonremunerat- ed voluntary donation is being promoted aggressively by local groups and such international partners of ours as the Red Cross. It is being based on the kinds of sociological data that identify the cultural values or other beliefs that could either support or mitigate against voluntary donation. The public education to increase this form of stocking a nation's blood supply has to be very culture specific, given the strong perceptions that surround blood in all societies. Most of the blood collected in Latin America and the Caribbean is still through replacement donation. Although this practice is being strongly discouraged, it is easy to see its genesis. There must be few more gratifying feelings than that of giving one's own blood not to go into some great amorphous pool but to help someone who is known and loved.

"It it critical that the appreciation of the importance of safe blood be seeded in the popular conscience. As the theme for World Health Day 2000 said so well, 'Safe blood starts with me'."

Of course, we must continue to promote testing of blood to prevent transmission of disease, and the Regional Program for External Evaluation of Performance on Serology for Transfusion-Transmitted Infections is a critical element in strengthening the capacity of the national laboratories. We have seen increased promotion of standards and norms for blood banks in the countries and sustained efforts to rationalize the large number of blood banks that are currently operating under a large number of different schemes in the countries. The support of the American Association of Blood Banks has been critical in establishing programs for standards. Because there is increased screening of blood, the number of transfusional infections has been reduced, and this has been documented very clearly in the case of Chagas' disease. I have been pleased to see the emphasis placed on education, even by distance, and the dissemination of both technical and popular information. It is critical that the appreciation of the importance of safe blood be seeded in the popular conscience. As the theme for World Health Day 2000 said so well, "Safe blood starts with me." 
The rationalization, licensing, and development of legislation for and monitoring of blood banks must be among the functions that have to fall within the "steering role" of the State, through the ministry of health. The State will have difficulty in operating all the blood banks needed, and, in reality, it should not do so. The success of nongovernmental organizations in this field has indeed been spectacular, as we see in the example of the American Red Cross in the United States. However, the monitoring of the conditions of collection, the use of standard protocols and equipment, and the capacities for screening of blood have to rest with or be expressly delegated by the State.

"There will always be the dream of developing a blood substitute, but for the forseeable future it will be necessary to ensure that this life-giving force is as safe as is humanly possible."

I have been gratified by the nature of PAHO's interprogrammatic approach to the provision of safe blood. Success in this area depends so much on collaboration between the groups and programs concerned with infectious disease, quality of care, and laboratory practice. And of course the provision of safe blood during disasters is one of the elements of preparedness that cannot be forgotten.

I believe that PAHO is on the correct path to assisting countries to have blood that is safe. There must, of course, never be complacency. There is no doubt that we will continue to have new infections affect humankind, and there will always be the challenge of determining whether these infections can or cannot be transmitted by blood transfusion. There will always be the dream of developing a blood substitute, but for the foreseeable future it will be necessary to ensure that this life-giving force is as safe as is humanly possible.

Perhaps the day will come when our poets will write differently of blood. When Shakespeare has Lady Macbeth say, "Make thick my blood," she is wishing for a steely resolve and courage to carry out her dastardly deed. In days to come, perhaps that expression may relate to giving a safe blood transfusion so as to enable someone to live and enjoy what life has to offer. But in the meantime we should all resolve to read carefully the contents of this special issue of the Revista/Journal and to see which of the excellent examples of good programs that it contains can be employed throughout our Region. I recommend it to you. 